\title{
Dual identities in fractional difference calculus within Riemann
}

Thabet Abdeljawad

\section{"Correspondence:}

thabet@cankaya.edu.tr

Department of Mathematics and

Computer Science, Çankaya

University, Ankara, 06530, Turkey

\begin{abstract}
We investigate two types of dual identities for Riemann fractional sums and differences. The first type relates nabla- and delta-type fractional sums and differences. The second type represented by the Q-operator relates left and right fractional sums and differences. These dual identities insist that in the definition of right fractional differences, we have to use both nabla and delta operators. The solution representation for a higher-order Riemann fractional difference equation is obtained as well.
\end{abstract}

Keywords: right (left) delta and nabla fractional sums; right (left) delta and nabla Riemann; Q-operator; dual identity

\section{Introduction}

During the last two decades, due to its widespread applications in different fields of science and engineering, fractional calculus has attracted the attention of many researchers [1-6].

Starting from the idea of discretizing the Cauchy integral formula, Miller and Ross [7] and Gray and Zhang [8] obtained discrete versions of left-type fractional integrals and derivatives, called fractional sums and differences. Fifteen years later, several authors started to deal with discrete fractional calculus [9-18], benefiting from the theory of time scales originated by Hilger in 1988 (see [19]).

In this article, we summarize some of the results mentioned in the above references and add more in the right-type and higher order fractional cases. Throughout the article, we almost agree with the previously presented definitions except for the definition of right fractional difference. We will figure out that these definitions seem to be more convenient than the previously presented ones by proving some dual identities. These identities fall into two kinds. The first kind relates nabla-type fractional differences and sums to delta ones. The second kind represented by the $Q$-operator relates left and right fractional sums and differences. This setting enables us to get identities resembling better the ordinary fractional case. Along with the previously mentioned points, we are able to fit a reasonable nabla integration by parts formula which remains in accordance with the one obtained in [15] but different from those obtained in [13] and [14]. The obtained dual identities are also used to obtain a delta integration by parts formula from the nabla one. The solution representation for the higher-order Riemann fractional difference equation is obtained as well and thus the result in [20] is generalized. We will see that the higherorder Riemann fractional difference initial value problem of non-integer order needs only

() 2013 Abdeljawad; licensee Springer. This is an Open Access article distributed under the terms of the Creative Commons Attribution License (http://creativecommons.org/licenses/by/2.0), which permits unrestricted use, distribution, and reproduction in any medium, provided the original work is properly cited. 
one initial condition which is not the case for the higher-order difference equation (i.e., of positive integer order).

The article is organized as follows. The remaining part of this section contains summary to some of the basic notations and definitions in delta and nabla calculus. Section 2 contains the definitions in the frame of delta and nabla fractional sums and differences in the Riemann sense. Moreover, some essential lemmas about the commutativity of the different fractional sum operators with the usual difference operators are established. These lemmas are vital to proceeding in the next sections. The third section contains some dual identities relating nabla and delta fractional sums and differences in the left and right cases. Using these dual identities, power formulas for nabla left and right fractional sums and a commutative law for nabla left and right sums are obtained. In Section 4 the integration by parts formula for nabla fractional sums and differences obtained in [20] is used by the help of the dual identities to obtain delta integration by parts formulas. Section 5 is devoted to higher-order initial fractional difference value problems into Riemann. Finally, in Section 6 the $Q$-operator is used to relate left and right fractional sums in nabla and delta case and hence relates delta and nabla Riemann fractional differences. The $Q$-dual identities obtained in this section expose the validity of the definition of delta and nabla right fractional differences where delta and nabla operators are used in each of the definitions of delta and nabla right fractional differences.

For a natural number $n$, the fractional polynomial is defined by

$$
t^{(n)}=\prod_{j=0}^{n-1}(t-j)=\frac{\Gamma(t+1)}{\Gamma(t+1-n)},
$$

where $\Gamma$ denotes the special gamma function, and the product is zero when $t+1-j=0$ for some $j$. More generally, for arbitrary $\alpha$, define

$$
t^{(\alpha)}=\frac{\Gamma(t+1)}{\Gamma(t+1-\alpha)},
$$

where we have the convention that division at pole yields zero. Given that the forward and backward difference operators are defined by

$$
\Delta f(t)=f(t+1)-f(t), \quad \nabla f(t)=f(t)-f(t-1)
$$

respectively, we define iteratively the operators $\Delta^{m}=\Delta\left(\Delta^{m-1}\right)$ and $\nabla^{m}=\nabla\left(\nabla^{m-1}\right)$, where $m$ is a natural number.

Here are some properties of the factorial function.

Lemma 1.1 [10] Assume the following factorial functions are well defined.

(i) $\Delta t^{(\alpha)}=\alpha t^{(\alpha-1)}$.

(ii) $(t-\mu) t^{(\mu)}=t^{(\mu+1)}$, where $\mu \in \mathbb{R}$.

(iii) $\mu^{(\mu)}=\Gamma(\mu+1)$.

(iv) If $t \leq r$, then $t^{(\alpha)} \leq r^{(\alpha)}$ for any $\alpha>r$.

(v) If $0<\alpha<1$, then $t^{(\alpha \nu)} \geq\left(t^{(\nu)}\right)^{\alpha}$.

(vi) $t^{(\alpha+\beta)}=(t-\beta)^{(\alpha)} t^{(\beta)}$. 
Also, for our purposes, we list down the following two properties, the proofs of which are straightforward.

$$
\begin{aligned}
& \nabla_{s}(s-t)^{(\alpha-1)}=(\alpha-1)(\rho(s)-t)^{(\alpha-2)}, \\
& \nabla_{t}(\rho(s)-t)^{(\alpha-1)}=-(\alpha-1)(\rho(s)-t)^{(\alpha-2)} .
\end{aligned}
$$

For the sake of the nabla fractional calculus, we have the following definition.

Definition 1.1 [19, 21-23]

(i) For a natural number $m$, the $m$ rising (ascending) factorial of $t$ is defined by

$$
t^{\bar{m}}=\prod_{k=0}^{m-1}(t+k), \quad t^{\overline{0}}=1 .
$$

(ii) For any real number, the $\alpha$ rising function is defined by

$$
t^{\bar{\alpha}}=\frac{\Gamma(t+\alpha)}{\Gamma(t)}, \quad t \in \mathbb{R}-\{\ldots,-2,-1,0\}, \quad 0^{\alpha}=0 .
$$

Regarding the rising factorial function, we observe the following:

(i)

$$
\nabla\left(t^{\bar{\alpha}}\right)=\alpha t^{\overline{\alpha-1}}
$$

(ii)

$$
\left(t^{\bar{\alpha}}\right)=(t+\alpha-1)^{(\alpha)} .
$$

(iii)

$$
\Delta_{t}(s-\rho(t))^{\bar{\alpha}}=-\alpha(s-\rho(t))^{\overline{\alpha-1}} .
$$

\section{Notation}

(i) For a real $\alpha>0$, we set $n=[\alpha]+1$, where $[\alpha]$ is the greatest integer less than $\alpha$.

(ii) For real numbers $a$ and $b$, we denote $\mathbb{N}_{a}=\{a, a+1, \ldots\}$ and ${ }_{b} \mathbb{N}=\{b, b-1, \ldots\}$.

(iii) For $n \in \mathbb{N}$ and real $a$, we denote

$$
{ }_{\ominus} \Delta^{n} f(t) \triangleq(-1)^{n} \Delta^{n} f(t)
$$

(iv) For $n \in \mathbb{N}$ and real $b$, we denote

$$
\nabla_{\Theta}^{n} f(t) \triangleq(-1)^{n} \nabla^{n} f(t)
$$

\section{Definitions and essential lemmas}

Definition 2.1 Let $\sigma(t)=t+1$ and $\rho(t)=t-1$ be forward and backward jumping operators, respectively. Then 
(i) The (delta) left fractional sum of order $\alpha>0$ (starting from $a$ ) is defined by

$$
\Delta_{a}^{-\alpha} f(t)=\frac{1}{\Gamma(\alpha)} \sum_{s=a}^{t-\alpha}(t-\sigma(s))^{(\alpha-1)} f(s), \quad t \in \mathbb{N}_{a+\alpha}
$$

(ii) The (delta) right fractional sum of order $\alpha>0$ (ending at $b$ ) is defined by

$$
\begin{aligned}
{ }_{b} \Delta^{-\alpha} f(t) & =\frac{1}{\Gamma(\alpha)} \sum_{s=t+\alpha}^{b}(s-\sigma(t))^{(\alpha-1)} f(s) \\
& =\frac{1}{\Gamma(\alpha)} \sum_{s=t+\alpha}^{b}(\rho(s)-t)^{(\alpha-1)} f(s), \quad t \in_{b-\alpha} \mathbb{N} .
\end{aligned}
$$

(iii) The (nabla) left fractional sum of order $\alpha>0$ (starting from $a$ ) is defined by

$$
\nabla_{a}^{-\alpha} f(t)=\frac{1}{\Gamma(\alpha)} \sum_{s=a+1}^{t}(t-\rho(s))^{\overline{\alpha-1}} f(s), \quad t \in \mathbb{N}_{a+1} .
$$

(iv) The (nabla) right fractional sum of order $\alpha>0$ (ending at $b$ ) is defined by:

$$
\begin{aligned}
{ }_{b} \nabla^{-\alpha} f(t) & =\frac{1}{\Gamma(\alpha)} \sum_{s=t}^{b-1}(s-\rho(t))^{\overline{\alpha-1}} f(s) \\
& =\frac{1}{\Gamma(\alpha)} \sum_{s=t}^{b-1}(\sigma(s)-t)^{\overline{\alpha-1}} f(s), \quad t \in_{b-1} \mathbb{N}
\end{aligned}
$$

Regarding the delta left fractional sum, we observe the following:

(i) $\Delta_{a}^{-\alpha}$ maps functions defined on $\mathbb{N}_{a}$ to functions defined on $\mathbb{N}_{a+\alpha}$.

(ii) $u(t)=\Delta_{a}^{-n} f(t), n \in \mathbb{N}$, satisfies the initial value problem

$$
\Delta^{n} u(t)=f(t), \quad t \in \mathbb{N}_{a}, \quad u(a+j-1)=0, \quad j=1,2, \ldots, n
$$

(iii) The Cauchy function $\frac{(t-\sigma(s))^{(n-1)}}{(n-1) !}$ vanishes at $s=t-(n-1), \ldots, t-1$.

Regarding the delta right fractional sum, we observe the following:

(i) ${ }_{b} \Delta^{-\alpha}$ maps functions defined on ${ }_{b} \mathbb{N}$ to functions defined on ${ }_{b-\alpha} \mathbb{N}$.

(ii) $u(t)={ }_{b} \Delta^{-n} f(t), n \in \mathbb{N}$, satisfies the initial value problem

$$
\nabla_{\ominus}^{n} u(t)=f(t), \quad t \in{ }_{b} \mathbb{N}, \quad u(b-j+1)=0, \quad j=1,2, \ldots, n .
$$

(iii) The Cauchy function $\frac{(\rho(s)-t)^{(n-1)}}{(n-1) !}$ vanishes at $s=t+1, t+2, \ldots, t+(n-1)$.

Regarding the nabla left fractional sum, we observe the following:

(i) $\nabla_{a}^{-\alpha}$ maps functions defined on $\mathbb{N}_{a}$ to functions defined on $\mathbb{N}_{a}$.

(ii) $\nabla_{a}^{-n} f(t)$ satisfies the $n$th order discrete initial value problem

$$
\nabla^{n} y(t)=f(t), \quad \nabla^{i} y(a)=0, \quad i=0,1, \ldots, n-1 .
$$

(iii) The Cauchy function $\frac{(t-\rho(s))^{\overline{n-1}}}{\Gamma(n)}$ satisfies $\nabla^{n} y(t)=0$. 
Regarding the nabla right fractional sum, we observe the following:

(i) ${ }_{b} \nabla^{-\alpha}$ maps functions defined on ${ }_{b} \mathbb{N}$ to functions defined on ${ }_{b} \mathbb{N}$.

(ii) ${ }_{b} \nabla^{-n} f(t)$ satisfies the $n$th order discrete initial value problem

$$
{ }_{\ominus} \Delta^{n} y(t)=f(t), \quad \ominus^{i} \Delta^{i}(b)=0, \quad i=0,1, \ldots, n-1 .
$$

The proof can be done inductively. Namely, assuming it is true for $n$, we have

$$
{ }_{\ominus} \Delta^{n+1}{ }_{b} \nabla^{-(n+1)} f(t)={ }_{\ominus} \Delta^{n}\left[-\Delta_{b} \nabla^{-(n+1)} f(t)\right] .
$$

By the help of (10), it follows that

$$
{ }_{\ominus} \Delta^{n+1}{ }_{b} \nabla^{-(n+1)} f(t)={ }_{\ominus} \Delta^{n}{ }_{b} \nabla^{-n} f(t)=f(t) .
$$

The other part is clear by using the convention that empty sums are taken to be 0 .

(iii) The Cauchy function $\frac{(s-\rho(t))^{\overline{n-1}}}{\Gamma(n)}$ satisfies $\ominus_{\ominus} \Delta^{n} y(t)=0$.

Definition 2.2 (i) [7] The (delta) left fractional difference of order $\alpha>0$ (starting from $a$ ) is defined by

$$
\Delta_{a}^{\alpha} f(t)=\Delta^{n} \Delta_{a}^{-(n-\alpha)} f(t)=\frac{\Delta^{n}}{\Gamma(n-\alpha)} \sum_{s=a}^{t-(n-\alpha)}(t-\sigma(s))^{(n-\alpha-1)} f(s), \quad t \in \mathbb{N}_{a+(n-\alpha)} .
$$

(ii) [15] The (delta) right fractional difference of order $\alpha>0$ (ending at $b$ ) is defined by

$$
{ }_{b} \Delta^{\alpha} f(t)=\nabla_{\ominus b}^{n} \Delta^{-(n-\alpha)} f(t)=\frac{(-1)^{n} \nabla^{n}}{\Gamma(n-\alpha)} \sum_{s=t+(n-\alpha)}^{b}(s-\sigma(t))^{(n-\alpha-1)} f(s), \quad t \in_{b-(n-\alpha)} \mathbb{N} .
$$

(iii) The (nabla) left fractional difference of order $\alpha>0$ (starting from $a$ ) is defined by

$$
\nabla_{a}^{\alpha} f(t)=\nabla^{n} \nabla_{a}^{-(n-\alpha)} f(t)=\frac{\nabla^{n}}{\Gamma(n-\alpha)} \sum_{s=a+1}^{t}(t-\rho(s))^{\overline{n-\alpha-1}} f(s), \quad t \in \mathbb{N}_{a+1} .
$$

(iv) The (nabla) right fractional difference of order $\alpha>0$ (ending at $b$ ) is defined by

$$
{ }_{b} \nabla^{\alpha} f(t)=\ominus \Delta^{n}{ }_{b} \nabla^{-(n-\alpha)} f(t)=\frac{(-1)^{n} \Delta^{n}}{\Gamma(n-\alpha)} \sum_{s=t}^{b-1}(s-\rho(t))^{\overline{n-\alpha-1}} f(s), \quad t \in{ }_{b-1} \mathbb{N} .
$$

Regarding the domains of the fractional type differences, we observe that

(i) The delta left fractional difference $\Delta_{a}^{\alpha}$ maps functions defined on $\mathbb{N}_{a}$ to functions defined on $\mathbb{N}_{a+(n-\alpha)}$.

(ii) The delta right fractional difference ${ }_{b} \Delta^{\alpha}$ maps functions defined on ${ }_{b} \mathbb{N}$ to functions defined on ${ }_{b-(n-\alpha)} \mathbb{N}$.

(iii) The nabla left fractional difference $\nabla_{a}^{\alpha}$ maps functions defined on $\mathbb{N}_{a}$ to functions defined on $\mathbb{N}_{a+n}$.

(iv) The nabla right fractional difference ${ }_{b} \nabla^{\alpha}$ maps functions defined on ${ }_{b} \mathbb{N}$ to functions defined on ${ }_{b-n} \mathbb{N}$. 
Lemma 2.1 [10] For any $\alpha>0$, the following equality holds:

$$
\Delta_{a}^{-\alpha} \Delta f(t)=\Delta \Delta_{a}^{-\alpha} f(t)-\frac{(t-a)^{\overline{\alpha-1}}}{\Gamma(\alpha)} f(a) .
$$

Lemma 2.2 [15] For any $\alpha>0$, the following equality holds:

$$
{ }_{b} \Delta^{-\alpha} \nabla_{\Theta} f(t)=\nabla_{\ominus b} \Delta^{-\alpha} f(t)-\frac{(b-t)^{\overline{\alpha-1}}}{\Gamma(\alpha)} f(b) .
$$

Lemma 2.3 [24] For any $\alpha>0$, the following equality holds:

$$
\nabla_{a+1}^{-\alpha} \nabla f(t)=\nabla \nabla_{a}^{-\alpha} f(t)-\frac{(t-a+1)^{\overline{\alpha-1}}}{\Gamma(\alpha)} f(a)
$$

The result of Lemma 2.3 was obtained in [24] by applying the nabla left fractional sum starting from $a$, not from $a+1$. Next, we will provide the version of Lemma 2.3 by applying the definition in this article. Actually, the nabla fractional sums defined in this article and those in [24] are related. For more details, we refer to [20].

Lemma 2.4 For any $\alpha>0$, the following equality holds:

$$
\nabla_{a}^{-\alpha} \nabla f(t)=\nabla \nabla_{a}^{-\alpha} f(t)-\frac{(t-a)^{\overline{\alpha-1}}}{\Gamma(\alpha)} f(a)
$$

Proof By the help of the following by parts identity:

$$
\begin{aligned}
\nabla_{s}\left[(t-s)^{\overline{\alpha-1}} f(s)\right] & =\nabla_{s}(t-s)^{\overline{\alpha-1}} f(s)+(t-\rho(s))^{\overline{\alpha-1}} \nabla_{s} f(s) \\
& =-(\alpha-1)(t-\rho(s))^{\overline{\alpha-2}} f(s)+(t-\rho(s))^{\overline{\alpha-1}} \nabla_{s} f(s),
\end{aligned}
$$

we have

$$
\begin{aligned}
\nabla_{a}^{-\alpha} \nabla f(t) & =\frac{1}{\Gamma(\alpha)} \sum_{s=a+1}^{t}(t-\rho(s))^{\overline{\alpha-1}} \nabla_{s} f(s) \\
& =\frac{1}{\Gamma(\alpha)}\left[\left.(t-s)^{\overline{\alpha-1}} f(s)\right|_{a} ^{t}+(\alpha-1) \sum_{s=a+1}^{t}(t-\rho(s))^{\overline{\alpha-2}} f(s)\right] \\
& =-\frac{(t-a)^{\overline{\alpha-1}}}{\Gamma(\alpha)} f(a)+\frac{1}{\Gamma(\alpha-1)} \sum_{s=a+1}^{t}(t-\rho(s))^{\overline{\alpha-2}} f(s) .
\end{aligned}
$$

On the other hand,

$$
\begin{aligned}
& \nabla \nabla_{a}^{-\alpha} f(t) \\
& \quad=\frac{1}{\Gamma(\alpha)} \sum_{s=a+1}^{t} \nabla_{t}(t-\rho(s))^{\overline{\alpha-1}} f(s)=\frac{1}{\Gamma(\alpha-1)} \sum_{s=a+1}^{t}(t-\rho(s))^{\overline{\alpha-2}} f(s) .
\end{aligned}
$$


Remark 2.1 Let $\alpha>0$ and $n=[\alpha]+1$. Then, by the help of Lemma 2.4, we have

$$
\nabla \nabla_{a}^{\alpha} f(t)=\nabla \nabla^{n}\left(\nabla_{a}^{-(n-\alpha)} f(t)\right)=\nabla^{n}\left(\nabla \nabla_{a}^{-(n-\alpha)} f(t)\right)
$$

or

$$
\nabla \nabla_{a}^{\alpha} f(t)=\nabla^{n}\left[\nabla_{a}^{-(n-\alpha)} \nabla f(t)+\frac{(t-a)^{\overline{n-\alpha-1}}}{\Gamma(n-\alpha)} f(a)\right]
$$

Then, using the identity

$$
\nabla^{n} \frac{(t-a)^{\overline{n-\alpha-1}}}{\Gamma(n-\alpha)}=\frac{(t-a)^{\overline{-\alpha-1}}}{\Gamma(-\alpha)}
$$

we infer that (26) is valid for any real $\alpha$.

By the help of Lemma 2.4, Remark 2.1 and the identity $\nabla(t-a)^{\overline{\alpha-1}}=(\alpha-1)(t-a)^{\overline{\alpha-2}}$, we arrive inductively at the following generalization.

Theorem 2.5 For any real number $\alpha$ and any positive integer $p$, the following equality holds:

$$
\nabla_{a}^{-\alpha} \nabla^{p} f(t)=\nabla^{p} \nabla_{a}^{-\alpha} f(t)-\sum_{k=0}^{p-1} \frac{(t-a)^{\overline{\alpha-p+k}}}{\Gamma(\alpha+k-p+1)} \nabla^{k} f(a),
$$

wheref is defined on $\mathbb{N}_{a}$ and some points before a.

Lemma 2.6 For any $\alpha>0$, the following equality holds:

$$
{ }_{b} \nabla^{-\alpha}{ }_{\ominus} \Delta f(t)={ }_{\ominus} \Delta_{b} \nabla^{-\alpha} f(t)-\frac{(b-t)^{\overline{\alpha-1}}}{\Gamma(\alpha)} f(b) .
$$

Proof By the help of the following discrete by parts formula:

$$
\begin{aligned}
\Delta_{s} & {\left[(\rho(s)-\rho(t))^{\overline{\alpha-1}} f(s)\right] } \\
& =(\alpha-1)(s-\rho(t))^{\overline{\alpha-2}} f(s)+(s-\rho(t))^{\overline{\alpha-1}} \Delta f(s),
\end{aligned}
$$

we have

$$
\begin{aligned}
{ }_{b} \nabla^{-\alpha}{ }_{a} \Delta f(t) \\
=-\frac{1}{\Gamma(\alpha)} \sum_{s=t}^{b-1}(s-\rho(t))^{\overline{\alpha-1}} \Delta f(s) \\
=\frac{1}{\Gamma(\alpha)}\left[-\sum_{s=t}^{b-1} \Delta_{s}\left((\rho(s)-\rho(t))^{\overline{\alpha-1}} f(s)\right)+(\alpha-1) \sum_{s=t}^{b-1}(s-\rho(t))^{\overline{\alpha-2}} f(s)\right] \\
=\frac{1}{\Gamma(\alpha-1)} \sum_{s=t}^{b-1}(s-\rho(t))^{\overline{\alpha-2}} f(s)-\frac{(b-t)^{\overline{\alpha-1}}}{\Gamma(\alpha)} f(b) .
\end{aligned}
$$


On the other hand,

$$
\begin{aligned}
& { }_{\ominus} \Delta_{b} \nabla^{-\alpha} f(t) \\
& =-\frac{1}{\Gamma(\alpha)} \sum_{s=t}^{b-1} \Delta_{t}(s-\rho(t))^{\overline{\alpha-1}} f(s)=\frac{1}{\Gamma(\alpha-1)} \sum_{s=t}^{b-1}(s-\rho(t))^{\overline{\alpha-2}} f(s),
\end{aligned}
$$

where the identity

$$
\Delta_{t}(s-\rho(t))^{\overline{\alpha-1}}=-(\alpha-1)(s-\rho(t))^{\overline{\alpha-2}}
$$

and the convention that $(0)^{\overline{\alpha-1}}=0$ are used.

Remark 2.2 Let $\alpha>0$ and $n=[\alpha]+1$. Then, by the help of Lemma 2.6, we can have

$$
{ }_{a} \Delta_{b} \nabla^{\alpha} f(t)={ }_{a} \Delta_{\odot} \Delta^{n}\left({ }_{b} \nabla^{-(n-\alpha)} f(t)\right)={ }_{\odot} \Delta^{n}\left({ }_{\odot} \Delta_{b} \nabla^{-(n-\alpha)} f(t)\right)
$$

or

$$
{ }_{\ominus} \Delta_{b} \nabla^{\alpha} f(t)={ }_{\ominus} \Delta^{n}\left[{ }_{b} \nabla^{-(n-\alpha)}{ }_{\ominus} \Delta f(t)+\frac{(b-t)^{\overline{n-\alpha-1}}}{\Gamma(n-\alpha)} f(b)\right] .
$$

Then, using the identity

$$
\ominus^{n} \frac{(b-t)^{\overline{n-\alpha-1}}}{\Gamma(n-\alpha)}=\frac{(b-t)^{\overline{-\alpha-1}}}{\Gamma(-\alpha)}
$$

we infer that (35) is valid for any real $\alpha$.

By the help of Lemma 2.6, Remark 2.2 and the identity $\Delta(b-t)^{\overline{\alpha-1}}=-(\alpha-1)(b-t)^{\overline{\alpha-2}}$, if we follow inductively, we arrive at the following generalization.

Theorem 2.7 For any real number $\alpha$ and any positive integer $p$, the following equality holds:

$$
{ }_{b} \nabla^{-\alpha}{ }_{\ominus} \Delta^{p} f(t)={ }_{\ominus} \Delta^{p}{ }_{b} \nabla^{-\alpha} f(t)-\sum_{k=0}^{p-1} \frac{(b-t)^{\overline{\alpha-p+k}}}{\Gamma(\alpha+k-p+1)} \ominus^{k} \Delta^{k} f(b),
$$

where $f$ is defined on ${ }_{b} \mathbb{N}$ and some points after $b$.

The following theorem modifies Theorem 2.5 when $f$ is only defined at $\mathbb{N}_{a}$.

Theorem 2.8 For any real number $\alpha$ and any positive integer $p$, the following equality holds:

$$
\nabla_{a+p-1}^{-\alpha} \nabla^{p} f(t)=\nabla^{p} \nabla_{a+p-1}^{-\alpha} f(t)-\sum_{k=0}^{p-1} \frac{(t-(a+p-1))^{\overline{\alpha-p+k}}}{\Gamma(\alpha+k-p+1)} \nabla^{k} f(a+p-1),
$$

where $f$ is defined on only $\mathbb{N}_{a}$. 
The proof follows by applying Remark 2.1 inductively.

Similarly, in the right case we have the following theorem.

Theorem 2.9 For any real number $\alpha$ and any positive integer $p$, the following equality holds:

$$
{ }_{b-p+1} \nabla^{-\alpha}{ }_{\ominus} \Delta^{p} f(t)={ }_{\ominus} \Delta^{p}{ }_{b-p+1} \nabla^{-\alpha} f(t)-\sum_{k=0}^{p-1} \frac{(b-p+1-t)^{\overline{\alpha-p+k}}}{\Gamma(\alpha+k-p+1)} \ominus^{k} f(b-p+1),
$$

where $f$ is defined on ${ }_{b} \mathbb{N}$ only.

\section{Dual identities for right fractional sums and differences}

The dual relations for left fractional sums and differences were investigated in [12]. Indeed, the following two lemmas are dual relations between the delta left fractional sums (differences) and the nabla left fractional sums (differences).

Lemma 3.1 [12] Let $0 \leq n-1<\alpha \leq n$ and let $y(t)$ be defined on $\mathbb{N}_{a}$. Then the following statements are valid.

(i) $\left(\Delta_{a}^{\alpha}\right) y(t-\alpha)=\nabla_{a}^{\alpha} y(t)$ for $t \in \mathbb{N}_{n+a}$.

(ii) $\left(\Delta_{a}^{-\alpha}\right) y(t+\alpha)=\nabla_{a}^{-\alpha} y(t)$ for $t \in \mathbb{N}_{a}$.

Lemma 3.2 [12] Let $0 \leq n-1<\alpha \leq n$ and let $y(t)$ be defined on $\mathbb{N}_{\alpha-n}$. Then the following statements are valid.

(i) $\Delta_{\alpha-n}^{\alpha} y(t)=\left(\nabla_{\alpha-n}^{\alpha} y\right)(t+\alpha)$ for $t \in \mathbb{N}_{-n}$.

(ii) $\Delta_{\alpha-n}^{-(n-\alpha)} y(t)=\left(\nabla_{\alpha-n}^{-(n-\alpha)} y\right)(t-n+\alpha)$ for $t \in \mathbb{N}_{0}$.

We remind that the above two dual lemmas for left fractional sums and differences were obtained when the nabla left fractional sum was defined by

$$
\nabla_{a}^{-\alpha} f(t)=\frac{1}{\Gamma(\alpha)} \sum_{s=a}^{t}(t-\rho(s))^{\overline{\alpha-1}} f(s), \quad t \in \mathbb{N}_{a}
$$

Now, in a way analogous to Lemma 3.1 and Lemma 3.2, for the right fractional summations and differences, we obtain the following lemmas.

Lemma 3.3 Let $y(t)$ be defined on ${ }_{b+1} \mathbb{N}$. Then the following statements are valid.

(i) $\left({ }_{b} \Delta^{\alpha}\right) y(t+\alpha)={ }_{b+1} \nabla^{\alpha} y(t)$ for $t \in{ }_{b-n} \mathbb{N}$.

(ii) $\left({ }_{b} \Delta^{-\alpha}\right) y(t-\alpha)={ }_{b+1} \nabla^{-\alpha} y(t)$ for $t \in{ }_{b} \mathbb{N}$.

Proof We prove only (i). The proof of (ii) is similar and easier.

$$
\begin{aligned}
\left({ }_{b} \Delta^{\alpha}\right) y(t+\alpha) & =(-1)^{n} \nabla^{n}{ }_{b} \Delta^{-(n-\alpha)} y(t+\alpha) \\
& =\frac{(-1)^{n} \nabla^{n}}{\Gamma(n-\alpha)} \sum_{s=t+n}^{b}(s-t-1-\alpha)^{(n-\alpha-1)} y(s) \\
& =\frac{(-1)^{n} \Delta^{n}}{\Gamma(n-\alpha)} \sum_{s=t}^{b}(s-t-1+n-\alpha)^{(n-\alpha-1)} y(s) .
\end{aligned}
$$


Using the identity $t^{\bar{\alpha}}=(t+\alpha-1)^{(\alpha)}$, we arrive at

$$
\begin{aligned}
\left({ }_{b} \Delta^{\alpha}\right) y(t+\alpha) & =\frac{(-1)^{n} \Delta^{n}}{\Gamma(n-\alpha)} \sum_{s=t}^{b}(s-\rho(t))^{\overline{n-\alpha-1}} y(s) \\
& =(-1)^{n} \Delta^{n}{ }_{b+1} \nabla^{-(n-\alpha)} y(t)={ }_{b+1} \nabla^{\alpha} y(t) .
\end{aligned}
$$

Lemma 3.4 Let $0 \leq n-1<\alpha \leq n$ and let $y(t)$ be defined on ${ }_{n-\alpha} \mathbb{N}$. Then the following statements are valid.

(i)

$$
{ }_{n-\alpha} \Delta^{\alpha} y(t)={ }_{n-\alpha+1} \nabla^{\alpha} y(t-\alpha), \quad t \in{ }_{n} \mathbb{N} .
$$

(ii)

$$
{ }_{n-\alpha} \Delta^{-(n-\alpha)} y(t)={ }_{n-\alpha+1} \nabla^{-(n-\alpha)} y(t+n-\alpha), \quad t \in{ }_{0} \mathbb{N} .
$$

Proof We prove (i), the proof of (ii) is similar. By the definition of right nabla difference, we have

$$
\begin{aligned}
{ }_{n-\alpha+1} \nabla^{\alpha} y(t-\alpha) & ={ }_{a} \Delta^{n} \frac{1}{\Gamma(n-\alpha)} \sum_{s=t-\alpha}^{n-\alpha}(s-\rho(t-\alpha))^{\overline{n-\alpha-1}} y(s) \\
& ={ }_{a} \Delta^{n} \frac{1}{\Gamma(n-\alpha)} \sum_{s=t-\alpha}^{n-\alpha}(s-\rho(t-\alpha))^{\overline{n-\alpha-1}} y(s) \\
& =\nabla_{b}^{n} \frac{1}{\Gamma(n-\alpha)} \sum_{s=t+n-\alpha}^{n-\alpha}(s-\rho(t+n-\alpha))^{\overline{n-\alpha-1}} y(s) .
\end{aligned}
$$

By using (9), it follows that

$$
{ }_{n-\alpha+1} \nabla^{\alpha} y(t-\alpha)=\nabla_{b}^{n} \frac{1}{\Gamma(n-\alpha)} \sum_{s=t+n-\alpha}^{n-\alpha}(s-\sigma(t))^{(n-\alpha-1)} y(s)={ }_{n-\alpha} \Delta^{\alpha} y(t) .
$$

Note that the above two dual lemmas for right fractional differences cannot be obtained if we apply the definition of the delta right fractional difference introduced in [14] and [13].

Lemma 3.5 [15] Let $\alpha>0, \mu>0$. Then

$$
{ }_{b-\mu} \Delta^{-\alpha}(b-t)^{(\mu)}=\frac{\Gamma(\mu+1)}{\Gamma(\mu+\alpha+1)}(b-t)^{(\mu+\alpha)} .
$$

The following commutative property for delta right fractional sums is Theorem 9 in [15].

Theorem 3.6 Let $\alpha>0, \mu>0$. Then, for all $t$ such that $t \equiv b-(\mu+\alpha)(\bmod 1)$, we have

$$
{ }_{b} \Delta^{-\alpha}\left[{ }_{b} \Delta^{-\mu} f(t)\right]={ }_{b} \Delta^{-(\mu+\alpha)} f(t)={ }_{b} \Delta^{-\mu}\left[{ }_{b} \Delta^{-\alpha} f(t)\right],
$$

where $f$ is defined on ${ }_{b} \mathbb{N}$. 
Proposition 3.7 Let $f$ be a real-valued function defined on ${ }_{b} \mathbb{N}$, and let $\alpha, \beta>0$. Then

$$
{ }_{b} \nabla^{-\alpha}\left[{ }_{b} \nabla^{-\beta} f(t)\right]={ }_{b} \nabla^{-(\alpha+\beta)} f(t)={ }_{b} \nabla^{-\beta}\left[{ }_{b} \nabla^{-\alpha} f(t)\right] .
$$

Proof The proof follows by applying Lemma 3.3(ii) and Theorem 3.6 above. Indeed,

$$
\begin{aligned}
{ }_{b} \nabla^{-\alpha}\left[{ }_{b} \nabla^{-\beta} f(t)\right] & ={ }_{b} \nabla^{-\alpha}{ }_{b-1} \Delta^{-\beta} f(t-\beta) \\
& ={ }_{b-1} \Delta^{-\alpha}{ }_{b-1} \Delta^{-\beta} f(t-(\alpha+\beta)) \\
& ={ }_{b-1} \Delta^{-(\alpha+\beta)} f(t-(\alpha+\mu))={ }_{b} \nabla^{-(\alpha+\beta)} y(t) .
\end{aligned}
$$

The following power rule for nabla right fractional differences plays an important role.

Proposition 3.8 Let $\alpha>0, \mu>-1$. Then, for $t \in{ }_{b} \mathbb{N}$, we have

$$
{ }_{b} \nabla^{-\alpha}(b-t)^{\bar{\mu}}=\frac{\Gamma(\mu+1)}{\Gamma(\alpha+\mu+1)}(b-t)^{\overline{\alpha+\mu}} .
$$

Proof By the dual formula (ii) of Lemma 3.3, we have

$$
{ }_{b} \nabla^{-\alpha}(b-t)^{\bar{\mu}}=\left.{ }_{b-1} \Delta^{-\alpha}(b-r)^{\bar{\mu}}\right|_{r=t-\alpha}=\frac{1}{\Gamma(\alpha)} \sum_{s=t}^{b-1}(s-t+\alpha-1)^{(\alpha-1)}(b-s)^{\bar{\mu}} .
$$

Then, by the identity $t^{\bar{\alpha}}=(t+\alpha-1)^{(\alpha-1)}$ and using the change of variable $r=s-\mu+1$, it follows that

$$
\begin{aligned}
{ }_{b} \nabla^{-\alpha}(b-t)^{\bar{\mu}} & =\frac{1}{\Gamma(\alpha)} \sum_{r=t-\mu+1}^{b-\mu}(r-\sigma(t-\alpha-\mu+1))^{(\alpha-1)}(b-r)^{\bar{\mu}} \\
& =\left.\left({ }_{b-\mu} \Delta^{-\alpha}(b-u)^{\bar{\mu}}\right)\right|_{u=-\alpha-\mu+1+t}
\end{aligned}
$$

which, by Lemma 3.5, leads to

$$
\begin{aligned}
{ }_{b} \nabla^{-\alpha}(b-t)^{\bar{\mu}} & =\frac{\Gamma(\mu+1)}{\Gamma(\alpha+\mu+1)}(b-t+\alpha+\mu-1)^{(\alpha+\mu)} \\
& =\frac{\Gamma(\mu+1)}{\Gamma(\alpha+\mu+1)}(b-t)^{\overline{\alpha+\mu}} .
\end{aligned}
$$

Similarly, for the nabla left fractional sum, we can have the following power formula and exponent law.

Proposition 3.9 Let $\alpha>0, \mu>-1$. Then, for $t \in \mathbb{N}_{a}$, we have

$$
\nabla_{a}^{-\alpha}(t-a)^{\bar{\mu}}=\frac{\Gamma(\mu+1)}{\Gamma(\alpha+\mu+1)}(t-a)^{\overline{\alpha+\mu}} .
$$

Proposition 3.10 Let $f$ be a real-valued function defined on $\mathbb{N}_{a}$, and let $\alpha, \beta>0$. Then

$$
\nabla_{a}^{-\alpha}\left[\nabla_{a}^{-\beta} f(t)\right]=\nabla_{a}^{-(\alpha+\beta)} f(t)=\nabla_{a}^{-\beta}\left[\nabla_{a}^{-\alpha} f(t)\right]
$$


Proof The proof can be achieved as in Theorem 2.1 [12], by expressing the left-hand side of (60), interchanging the order of summation and using the power formula (59). Alternatively, the proof can be done by following the proof of Proposition 3.7 with the help of the dual formula for left fractional sum in Lemma 3.1 after its arrangement according to our definitions.

\section{Integration by parts for fractional sums and differences}

In this section we state the integration by parts formulas for nabla fractional sums and differences obtained in [20], then use the dual identities to obtain delta integration by part formulas.

Proposition 4.1 [20] For $\alpha>0, a, b \in \mathbb{R}, f$ defined on $\mathbb{N}_{a}$ and $g$ defined on ${ }_{b} \mathbb{N}$, we have

$$
\sum_{s=a+1}^{b-1} g(s) \nabla_{a}^{-\alpha} f(s)=\sum_{s=a+1}^{b-1} f(s)_{b} \nabla^{-\alpha} g(s) .
$$

Proof By the definition of the nabla left fractional sum, we have

$$
\sum_{s=a+1}^{b-1} g(s) \nabla_{a}^{-\alpha} f(s)=\frac{1}{\Gamma(\alpha)} \sum_{s=a+1}^{b-1} g(s) \sum_{r=a+1}^{s}(s-\rho(r))^{\overline{\alpha-1}} f(r) .
$$

If we interchange the order of summation, we reach (61).

By the help of Theorem 2.5, Proposition 3.10, (17) and that $\nabla_{a}^{-(n-\alpha)} f(a)=0$, the authors in [20] obtained the following left important tools which led to a nabla integration by parts formula for fractional differences.

Proposition 4.2 [20] For $\alpha>0$ and $f$ defined in a suitable domain $\mathbb{N}_{a}$, we have

$$
\begin{aligned}
& \nabla_{a}^{\alpha} \nabla_{a}^{-\alpha} f(t)=f(t), \\
& \nabla_{a}^{-\alpha} \nabla_{a}^{\alpha} f(t)=f(t), \quad \text { when } \alpha \notin \mathbb{N},
\end{aligned}
$$

and

$$
\nabla_{a}^{-\alpha} \nabla_{a}^{\alpha} f(t)=f(t)-\sum_{k=0}^{n-1} \frac{(t-a)^{\bar{k}}}{k !} \nabla^{k} f(a), \quad \text { when } \alpha=n \in \mathbb{N} .
$$

By the help of Theorem 2.7, Proposition 3.7, (18) and that ${ }_{b} \nabla^{-(n-\alpha)} f(b)=0$, the authors in [20] also obtained the following right important tool.

Proposition 4.3 [20] For $\alpha>0$ and $f$ defined in a suitable domain ${ }_{b} \mathbb{N}$, we have

$$
\begin{aligned}
& { }_{b} \nabla^{\alpha}{ }_{b} \nabla^{-\alpha} f(t)=f(t), \\
& { }_{b} \nabla^{-\alpha}{ }_{b} \nabla^{\alpha} f(t)=f(t), \quad \text { when } \alpha \notin \mathbb{N},
\end{aligned}
$$

and

$$
{ }_{b} \nabla^{-\alpha}{ }_{b} \nabla^{\alpha} f(t)=f(t)-\sum_{k=0}^{n-1} \frac{(b-t)^{\bar{k}}}{k !} \ominus_{\ominus} \Delta^{k} f(b), \quad \text { when } \alpha=n \in \mathbb{N} \text {. }
$$


Proposition 4.4 [20] Let $\alpha>0$ be non-integer and $a, b \in \mathbb{R}$ such that $a<b$ and $b \equiv$ $a(\bmod 1)$. Iff is defined on ${ }_{b} \mathbb{N}$ and $g$ is defined on $\mathbb{N}_{a}$, then

$$
\sum_{s=a+1}^{b-1} f(s) \nabla_{a}^{\alpha} g(s)=\sum_{s=a+1}^{b-1} g(s)_{b} \nabla^{\alpha} f(s)
$$

The proof was achieved by making use of Proposition 4.1 and the tools of Proposition 4.2 and Proposition 4.3.

Now, by the above nabla integration by parts formulas and the dual identities in Lemma 3.1 adjusted to our definitions and Lemma 3.3, we can obtain delta integration by parts formulas.

Proposition 4.5 Let $\alpha>0, a, b \in \mathbb{R}$ such that $a<b$ and $b \equiv a(\bmod 1)$. Iff is defined on $\mathbb{N}_{a}$ and $g$ is defined on ${ }_{b} \mathbb{N}$, then we have

$$
\sum_{s=a+1}^{b-1} g(s)\left(\Delta_{a+1}^{-\alpha} f\right)(s+\alpha)=\sum_{s=a+1}^{b-1} f(s)_{b-1} \Delta^{-\alpha} g(s-\alpha) .
$$

Proposition 4.6 Let $\alpha>0$ be non-integer and assume that $b \equiv a(\bmod 1)$. Iff is defined on ${ }_{b} \mathbb{N}$ and $g$ is defined on $\mathbb{N}_{a}$, then

$$
\sum_{s=a+1}^{b-1} f(s) \Delta_{a+1}^{\alpha} g(s-\alpha)=\sum_{s=a+1}^{b-1} g(s)_{b-1} \Delta^{\alpha} f(s+\alpha) .
$$

\section{Higher-order fractional difference initial value problem within Riemann}

Let $\alpha>0$ be non-integer, $n=[\alpha]+1$ and $a(\alpha)=a+n-1$. Consider the fractional initial difference equation

$$
\begin{aligned}
& \nabla_{a(\alpha)-1}^{\alpha} y(t)=f(t, y(t)), \quad t=a(\alpha)+1, a(\alpha)+2, \ldots \\
& \left.\nabla_{a(\alpha)-1}^{-(n-\alpha)} y(t)\right|_{t=a(\alpha)}=y(a(\alpha))=c .
\end{aligned}
$$

Apply the sum operator $\nabla_{a(\alpha)}^{-\alpha}$ to both sides of (72) to get

$$
\nabla_{a(\alpha)}^{-\alpha}\left\{\nabla_{a(\alpha)}^{\alpha} y(t)+\nabla^{n} \frac{(t-a(\alpha)+1)^{\overline{n-\alpha-1}}}{\Gamma(n-\alpha)} y(a(\alpha))\right\}=\nabla_{a(\alpha)}^{-\alpha} f(t, y(t)) .
$$

Applying (64), we reach

$$
y(t)+\nabla_{a(\alpha)}^{-\alpha} \nabla^{n} \frac{(t-a(\alpha)+1)^{\overline{n-\alpha-1}}}{\Gamma(n-\alpha)} y(a(\alpha))=\nabla_{a(\alpha)}^{-\alpha} f(t, y(t)) .
$$

Now, set $g_{\alpha}(t)=\frac{(t-a(\alpha)+1)^{\overline{n-\alpha-1}}}{\Gamma(n-\alpha)} y(a(\alpha))$, then by Theorem 2.8 with $p=n$ and $f(t)=g_{\alpha}(t)$, we have

$$
\nabla_{a(\alpha)}^{-\alpha} \nabla^{n} g_{\alpha}(t)=\nabla^{n} \nabla_{a(\alpha)}^{-\alpha} g_{\alpha}(t)-\sum_{k=0}^{n-1} \frac{(t-a(\alpha))^{\overline{\alpha-n+k}}}{\Gamma(\alpha+k-n+1)} \nabla^{k} g_{\alpha}(a(\alpha)) .
$$


Noting that $\nabla^{k} g_{\alpha}(a(\alpha))=y(a(\alpha))$ for $k=0,1, \ldots, n-1$, by the power formula (59), we conclude that

$$
\nabla_{a(\alpha)}^{-\alpha} \nabla^{n} g_{\alpha}(t)=0-\nabla^{n} \frac{(t-a(\alpha)+1)^{\overline{\alpha-1}} y(a(\alpha))}{\Gamma(\alpha)}-\sum_{k=0}^{n-1} \frac{(t-a(\alpha))^{\overline{\alpha-n+k}}}{\Gamma(\alpha+k-n+1)} y(a(\alpha)) .
$$

Then the substitution in (74) will lead to the following solution representation:

$$
y(t)=\nabla^{n} \frac{(t-a(\alpha)+1)^{\overline{\alpha-1}} y(a(\alpha))}{\Gamma(\alpha)}+\sum_{k=0}^{n-1} \frac{(t-a(\alpha))^{\overline{\alpha-n+k}}}{\Gamma(\alpha+k-n+1)} c+\nabla_{a(\alpha)}^{-\alpha} f(t, y(t)) .
$$

As a particular case, if $0<\alpha<1$, then $a(\alpha)=a$ and hence

$$
y(t)=\frac{(t-a+1)^{\overline{\alpha-1}}}{\Gamma(\alpha)} y(a)+\nabla_{a}^{-\alpha} f(t, y(t)),
$$

which is the result obtained in [20].

If $1<\alpha<2$, then $n=2$ and $a(\alpha)=a+1$ and the initial value problem (72) becomes

$$
\begin{aligned}
& \nabla_{a}^{\alpha} y(t)=f(t, y(t)), \quad t=a+2, a+3, \ldots, \\
& \left.\nabla_{a}^{-(2-\alpha)} y(t)\right|_{t=a+1}=y(a+1)=c .
\end{aligned}
$$

From (77), the solution is

$$
y(t)=\frac{(t-a)^{\overline{\alpha-3}} c}{\Gamma(\alpha-2)}+\frac{(t-a-1)^{\overline{\alpha-2}} c}{\Gamma(\alpha-1)}+\frac{(t-a-1)^{\overline{\alpha-1}} c}{\Gamma(\alpha)}+\nabla_{a+1}^{-\alpha} f(t, y(t)) .
$$

Combining the first two terms then

$$
y(t)=\frac{(t-a)^{\overline{\alpha-2}} c}{\Gamma(\alpha-1)}+\frac{(t-a-1)^{\overline{\alpha-1}} c}{\Gamma(\alpha)}+\nabla_{a+1}^{-\alpha} f(t, y(t)) .
$$

Again combining, we reach

$$
y(t)=\frac{(t-a)^{\overline{\alpha-1}} c}{\Gamma(\alpha)}+\nabla_{a+1}^{-\alpha} f(t, y(t)) .
$$

If we proceed inductively, we can state the following.

Theorem 5.1 Let $\alpha>0$ be non-integer, $n=[\alpha]+1$ and $a(\alpha)=a+n-1$. Then the solution of the fractional initial difference equation

$$
\begin{aligned}
& \nabla_{a(\alpha)-1}^{\alpha} y(t)=f(t, y(t)), \quad t=a(\alpha)+1, a(\alpha)+2, \ldots, \\
& \left.\nabla_{a(\alpha)-1}^{-(n-\alpha)} y(t)\right|_{t=a(\alpha)}=y(a(\alpha))=c,
\end{aligned}
$$

is given by

$$
y(t)=\frac{(t-a(\alpha)+1)^{\overline{\alpha-1}} c}{\Gamma(\alpha)}+\nabla_{a(\alpha)}^{-\alpha} f(t, y(t)) .
$$


The surprise in Theorem 5.1 is that the higher-order Riemann fractional difference initial value problem of non-integer order needs only one initial condition which is not the case for the higher-order difference equation (i.e., of positive integer order). Also, the solution consists of two terms, which is not the case for fractional Cauchy problems into Riemann.

\section{The $\mathbf{Q}$-operator and fractional difference equations}

If $f(s)$ is defined on $\mathbb{N}_{a} \cap{ }_{b} \mathbb{N}$ and $a \equiv b(\bmod 1)$, then $(Q f)(s)=f(a+b-s)$. The $Q$-operator generates a dual identity by which the left-type and the right-type fractional sums and differences are related. Using the change of variable $u=a+b-s$, in [9] it was shown that

$$
\Delta_{a}^{-\alpha} Q f(t)=Q_{b} \Delta^{-\alpha} f(t)
$$

and hence

$$
\Delta_{a}^{\alpha} Q f(t)=\left(Q_{b} \Delta^{\alpha} f\right)(t)
$$

The proof of (86) follows by the definition, (85) and by noting that

$$
-Q \nabla f(t)=\Delta Q f(t)
$$

Similarly, in the nabla case, we have

$$
\nabla_{a}^{-\alpha} Q f(t)=Q_{b} \nabla^{-\alpha} f(t)
$$

and hence

$$
\nabla_{a}^{\alpha} Q f(t)=\left(Q_{b} \nabla^{\alpha} f\right)(t)
$$

The proof of (88) follows by the definition, (87) and that

$$
-Q \Delta f(t)=\nabla Q f(t)
$$

It is remarkable to mention that the $Q$-dual identity (86) cannot be obtained if the definition of the delta right fractional difference introduced by Bastos et al. in [14] or by Atıc et al. in [13] is used. Thus, the definition introduced in [9] and [15] is more convenient. Analogously, the Q-dual identity (88) indicates that the nabla right Riemann fractional differences presented in this article are also more convenient.

It is clear from the above argument that the $Q$-operator agrees with its continuous counterpart when applied to left and right fractional Riemann integrals Riemann derivatives. More generally, this discrete version of the $Q$-operator can be used to transform the discrete delay-type fractional functional difference dynamic equations to advanced ones. For details in the continuous counterparts, see [25].

Competing interests

The author declares that he has no competing interests. 


\section{References}

1. Podlubny, I: Fractional Differential Equations. Academic Press, San Diego (1999)

2. Samko, G, Kilbas, AA, Marichev, Ol: Fractional Integrals and Derivatives: Theory and Applications. Gordon \& Breach, Yverdon (1993)

3. Kilbas, A, Srivastava, MH, Trujillo, JJ: Theory and Application of Fractional Differential Equations. North Holland Mathematics Studies, vol. 204 (2006)

4. Agrawal, OP, Baleanu, D: A Hamiltonian formulation and a direct numerical scheme for fractional optimal control problems. J. Vib. Control 13(9-10), 1269-1281 (2007)

5. Scalas, E: Mixtures of compound Poisson processes as models of tick-by-tick financial data. Chaos Solitons Fractals 34(1), 33-40 (2007)

6. Baleanu, D, Trujillo, JJ: On exact solutions of a class of fractional Euler-Lagrange equations. Nonlinear Dyn. 52(4) 331-335 (2008)

7. Miller, KS, Ross, B: Fractional difference calculus. In: Proceedings of the International Symposium on Univalent Functions, Fractional Calculus and Their Applications, Nihon University, Koriyama, Japan, pp. 139-152 (1989)

8. Gray, HL, Zhang, NF: On a new definition of the fractional difference. Math. Comput. 50(182), 513-529 (1988)

9. Abdeljawad, T: On Riemann and Caputo fractional differences. Comput. Math. Appl. 62(3), 1602-1611 (2011)

10. Atıcı, FM, Eloe, PW: A transform method in discrete fractional calculus. Int. J. Differ. Equ. 2(2), 165-176 (2007)

11. Atıcl, FM, Eloe, PW: Initial value problems in discrete fractional calculus. Proc. Am. Math. Soc. 137, 981-989 (2009)

12. AtıCl, FM, Eloe, PW: Discrete fractional calculus with the nabla operator. Electron. J. Qual. Theory Differ. Equ. 2009, 3 (2009) (Spec. Ed. I)

13. AtıCı, FM, Şengül, S: Modelling with fractional difference equations. J. Math. Anal. Appl. 369, 1-9 (2010)

14. Bastos, NRO, Ferreira, RAC, Torres, DFM: Discrete-time fractional variational problems. Signal Process. 91(3), 513-524 (2011)

15. Abdeljawad, T, Baleanu, D: Fractional differences and integration by parts. J. Comput. Anal. Appl. 13(3), 574-582 (2011)

16. Anastassiou, GA: Principles of delta fractional calculus on time scales and inequalities. Math. Comput. Model. $\mathbf{5 2 ,}$ 556-566 (2010)

17. Anastassiou, GA: Nabla discrete calculus and nabla inequalities. Math. Comput. Model. 51, 562-571 (2010)

18. Anastassiou, GA: Foundations of nabla fractional calculus on time scales and inequalities. Comput. Math. Appl. 59 3750-3762 (2010)

19. Bohner, M, Peterson, A: Advances in Dynamic Equations on Time Scales. Birkhäuser, Boston (2003)

20. Abdeljawad, T, Atıcı, F: On the definitions of nabla fractional operators. Abstr. Appl. Anal. 2012, Article ID 406757 (2012). doi:10.1155/2012/406757

21. Boros, G, Moll, V: Irresistible Integrals: Symbols, Analysis and Experiments in the Evaluation of Integrals. Cambridge University Press, Cambridge (2004)

22. Graham, RL, Knuth, DE, Patashnik, O: Concrete Mathematics. A Foundation for Computer Science, 2nd edn. Addison-Wesley, Reading (1994)

23. Spanier, J, Oldham, KB: The Pochhammer Polynomials $(x)_{n}$. An Atlas of Functions, pp. 149-156. Hemisphere, Washington (1987)

24. Atıcl, FM, Eloe, PW: Gronwall's inequality on discrete fractional calculus. Comput. Math. Appl. 64(10), 3193-3200 (2012). doi:10.1016/j.camwa.2011.11.029

25. Abdeljawad Maraaba, T, Baleanu, D, Jarad, F: Existence and uniqueness theorem for a class of delay differential equations with left and right Caputo fractional derivatives. J. Math. Phys. 49, Article ID 083507 (2008)

doi:10.1186/1687-1847-2013-36

Cite this article as: Abdeljawad: Dual identities in fractional difference calculus within Riemann. Advances in Difference Equations 2013 2013:36.

\section{Submit your manuscript to a SpringerOpen ${ }^{\circ}$ journal and benefit from:}

- Convenient online submission

Rigorous peer review

- Immediate publication on acceptance

- Open access: articles freely available online

- High visibility within the field

- Retaining the copyright to your article 